\title{
Mycobacteriosis in a Domestic Ferret (Mustela putorius furo)
}

\author{
Makoto NAKATA $^{1) *}$, Yasutsugu MIWA ${ }^{1)}$, Masaya TSUBOI'²) and Kazuyuki UCHIDA²) \\ 1) Miwa Exotic Animal Hospital, 1-25-5 Komagome, Toshima-ku, Tokyo 170-0003, Japan \\ ${ }^{2)}$ Laboratory of Veterinary Pathology, Graduate School of Agricultural and Life Sciences, The University of Tokyo, 1-1-1 Yayoi, Bunkyo- \\ ku, Tokyo 113-8657, Japan
}

(Received 18 October 2013/Accepted 25 December 2013/Published online in J-STAGE 10 January 2014)

ABSTRACT. A 4-year-old spayed female ferret presented with a 2-month history of anorexia, vomiting and occasional diarrhea. Abdominal ultrasonography revealed thickening of the gastric wall and enlarged abdominal lymph nodes. Biopsy samples from the thickened gastric wall, enlarged abdominal lymph nodes and liver were taken during an exploratory laparotomy. Based on the histopathological examination, mycobacterium infection was diagnosed. The bacterial species could not be identified by additional diagnostic tests of feces, including fecal smear, culture and polymerase chain reaction (PCR). The ferret was treated with prednisolone and multiple antimicrobials, including rifampicin, azithromycin and enrofloxacin, but did not improve with treatment and died 220 days after the first presentation.

KEY WORDS: ferret, mycobacterium infection.

doi: 10.1292/jvms.13-0521; J. Vet. Med. Sci. 76(5): 705-709, 2014

Ferrets (Mustela putorius furo) are susceptible to a variety of mycobacterial infections [2, 5, 7, 16, 18]. Mycobacteriosis is one of the most important diseases of feral ferrets in New Zealand. Ferrets were originally released in New Zealand in the 1880 s to help control rabbit populations, and the number of feral ferrets is large $[2,3,10,14]$. Compared with other feral carnivores in New Zealand, only ferrets have a high incidence of mycobacteriosis [4, 9, 10, 14]. The most commonly reported infecting species is Mycobacterium bovis; however, M. avium complex (MAC) and M. triplex are also frequently isolated in New Zealand [4]. Reports of mycobacteriosis in domestic ferrets are sporadic $[6,7,11$, $13,15,16,18]$ in other parts of the world. In Japan, the only report of mycobacteriosis in a domestic ferret was presented as a case report of suspected mycobacteriosis at an academic conference (The 29th Annual Meeting of Japanese Society of Clinical Veterinary Medicine).

Ferrets with mycobacterial infections can present with a variety of clinical signs, such as anorexia, emaciation, vomiting, diarrhea, lymph node enlargement, conjunctival lesions, splenomegaly and pneumonia; the predominant clinical signs vary depending on the species of mycobacteria. Mycobacteriosis in ferrets most commonly affects the gastrointestinal tract and/or liver [1, 14]. It has been postulated that mycobacterium infections are associated with the feeding of raw meat and poultry and unpasteurized dairy products $[2,16]$.

The present report describes a ferret with vomiting and anorexia due to a mycobacteriosis infection, and this report

*Correspondence to: Nakata, M., Miwa Exotic Animal Hospital, 1-25-5 Komagome, Toshima-ku, Tokyo 170-0003, Japan.

e-mail: azu05pooh@hotmail.com

(C)2014 The Japanese Society of Veterinary Science

This is an open-access article distributed under the terms of the Creative Commons Attribution Non-Commercial No Derivatives (by-nc-nd) License $<$ http://creativecommons.org/licenses/by-nc-nd/3.0/>. presents the first detailed case of mycobacteriosis in a domestic ferret in Japan.

A 4-year-old spayed female ferret presented with a 2-month history of anorexia, vomiting and occasional diarrhea. The ferret was kept indoors and fed commercial ferret foods. On the first admission to our clinic, mild emaciation and nausea were observed. The nictitating membrane of the right eye had been protruded for several months. Abdominal palpation revealed a mass lesion located in the cranial abdomen. There were no significant changes on a complete blood cell (CBC) count, but mild hypoalbuminemia $(2.3 \mathrm{~g} / \mathrm{d} l$; reference value, $2.5-4.0 \mathrm{~g} / \mathrm{d} l)$, mild hyponatremia $(127 \mathrm{mEq} / l$; reference value, $142-148 \mathrm{mEq} / l$ ) and mild hypoglycemia ( $72 \mathrm{mg} / \mathrm{d} l$; reference value, $80-117 \mathrm{mg} / \mathrm{d} l$ ) were detected on serum biochemistry analysis. Thoracic and abdominal radiographs revealed gas retention in the stomach and intestines, and a barium study of the gastrointestinal tract demonstrated a slightly delayed gastric emptying time. Abdominal ultrasonography revealed thickening of the gastric wall and abdominal lymphadenomegaly (Fig. 1A). On the basis of these findings, a presumptive diagnosis of gastrointestinal tumor was considered, and an exploratory laparotomy was performed.

At the exploratory laparotomy, a thickened and indurated gastric wall was found at approximately half of the greater curvature. The perigastric and mesenteric lymph nodes were enlarged (Fig. 1B). The liver appeared yellowish in color, and white spots were disseminated throughout all lobes. Biopsy specimens were taken from the stomach, abdominal lymph nodes and liver and were fixed in $10 \%$ neutral buffered formalin. Since no other abnormal findings were observed in other organs, including the adrenal glands and pancreas, the operation was finished in a routine manner. Tissue samples were embedded in paraffin, cut into sections of $4 \mu \mathrm{m}$ and stained with hematoxylin and eosin (HE) for routine histological examination and with Ziehl-Neelsen for the detection of acid-fast bacteria. Imprint smears of the 


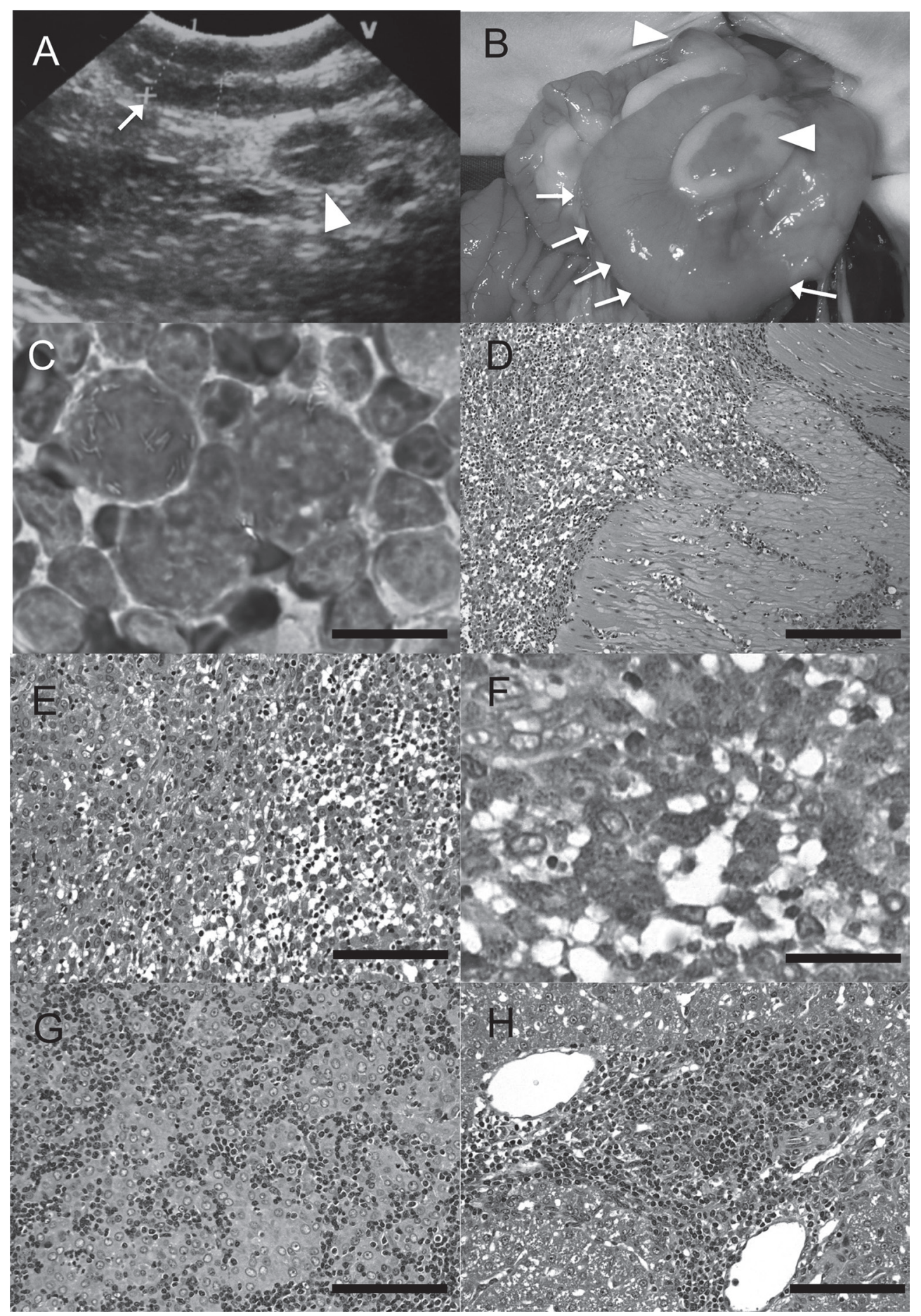

excised tissues were made and stained with Wright-Giemsa. Cytologic evaluation of impression smears from the stomach and lymph nodes identified numerous macrophages containing rod-shaped to filamentous nonstaining bacterial organisms in their cytoplasm (Fig. 1C). Histopathologically, there was severe multifocal to diffuse infiltration of inflammatory cells, consisting mainly of macrophages and epithelioid cells with a small number of lymphocytes and neutrophils, throughout the lamina propria into the muscular layer (Fig. 1D and 1E). The macrophages and epithelioid cells contained intracytoplasmic filamentous, pale to slightly basophilic bacteria, which were intensely positive with Ziehl-Neelsen stain (Fig. 1F). Similar granulomatous lesions were also observed in the lymph node and liver. In the lymph 
Table 1. The results of white blood cell (WBC) counts and packed cell volume (PCV) during treatment. Blood transfusion was performed on the 194th day

\begin{tabular}{|c|c|c|c|c|c|c|c|}
\hline \multirow[t]{2}{*}{ Day } & \multicolumn{6}{|c|}{$\begin{array}{c}\mathrm{WBC}(/ \mu l) \\
(2,500-8,500)^{*}\end{array}$} & \multirow[t]{2}{*}{$\begin{array}{l}\mathrm{PCV}(\%) \\
(46-57)^{*}\end{array}$} \\
\hline & Total & Neutrophils & Lymphocytes & Monocytes & Eosinophils & Basophils & \\
\hline 0 & 4,300 & ND & ND & ND & ND & ND & 38 \\
\hline 50 & 3,600 & ND & ND & ND & ND & ND & 29 \\
\hline 120 & 700 & 504 & 182 & 14 & 0 & 0 & 11 \\
\hline 127 & 1,200 & 768 & 264 & 144 & 24 & 0 & 10 \\
\hline 141 & 1,200 & ND & ND & ND & ND & ND & 15 \\
\hline 175 & 1,400 & 1,148 & 168 & 84 & 0 & 0 & 22 \\
\hline
\end{tabular}

*: Reference value, ND: Not Done

node, epithelioid macrophages containing acid-fast bacterial organisms diffusely infiltrated the sinus (Fig. 1G). In the liver, there was mild to moderate accumulation of macrophages and lymphocytes with a few neutrophils around the portal area. The macrophages in these lesions also contained intracytoplasmic acid-fast bacterial organisms (Fig. 1H). Based on the findings from cytological and histopathological examinations, mycobacterium infection was diagnosed.

To confirm the presence of mycobacterium in feces, a fecal smear evaluation, mycobacterial culture and polymerase chain reaction (PCR) were performed by a commercial laboratory (Kotobiken Medical Laboratories, Tokyo, Japan). Positive results were obtained from microscopic evaluation of a fecal smear stained with Ziehl-Neelsen stain (Gaffky 5; range, 1-10) and from a fluorescence method (2+; range, 1-3). Mycobacterium culture using Ogawa $\mathrm{K}$ medium was negative at 4, 8 and 12 weeks of culture. PCR was performed to identify the Mycobacterium tuberculosis complex (M. tuberculosis, M. bovis, M. microti and M. africans) and MAC. All PCR results were negative, which therefore excluded both the M. tuberculosis complex and the MAC. Ultimately, the mycobacterial species could not be definitively identified.

The ferret was treated with a regimen of rifampicin (10 $\mathrm{mg} / \mathrm{kg}$ twice a day; Rifadin ${ }^{\circledR}$; Daiichisankyo, Tokyo, Japan), azithromycin $\left(10 \mathrm{mg} / \mathrm{kg}\right.$ twice a day; Zithromax ${ }^{\circledR}$; Pfizer, Tokyo, Japan), enrofloxacin (5 mg/kg once a day; Baytril ${ }^{\circledR}$; Bayer, Osaka, Japan) and prednisolone $(0.25-1 \mathrm{mg} / \mathrm{kg}$ once a day; Prednisolone; Takeda, Osaka, Japan); other medicines for hepatic and intestinal disorders were also administered. The ferret did not significantly improve with treatment and developed progressive anemia and leukopenia in addition to icterus, severe splenomegaly and ascites. In a blood smear examination, the anemia appeared mildly regenerative, and there were no other erythrocyte morphologic abnormalities or atypical white blood cells (WBC). The results of WBC counts and packed cell volume (PCV) during treatment are shown in Table 1. Further diagnostic procedures, such as splenectomy and bone marrow aspiration, were declined by the owner. The ferret's condition improved slightly following a blood transfusion (on day 194), hematopoietic stimulants, such as erythropoietin and supportive therapies, such as forced feeding and hypodermoclysis. Despite treatment, the ferret gradually weakened and died 220 days after the initial presentation. Necropsy was not performed.

Mycobacteriosis is diagnosed by results of tissue biopsy, including acid-fast staining, PCR testing and bacterial culture. The most commonly reported mycobacterium infections in ferrets are caused by M. bovis and MAC $[2,5,14]$. However, MAC and the M. tuberculosis complex, which includes $M$. bovis, an important atypical mycobacterium in human medicine, were not identified in this patient's feces. Although it was unknown whether the mycobacteria in the lesions were the same species detected in the feces because we were unable to perform PCR with samples from the lesions, and the testable species of Mycobacterium are limited in commercial laboratories, it is possible that the mycobacterium strain microscopically identified in this case is a rare species.

Choice of and response to treatment vary depending on the species of mycobacterium $[2,7,11,13,15,16,18,19]$. Macrolides, including clarithromycin and azithromycin, are

Fig.1. (A) Abdominal ultrasonography. Thickening of the gastric wall (arrow) and swelling of the abdominal lymph nodes (arrowhead) are present. (B) Exploratory laparotomy. A thickened and indurated gastric wall is found at the approximate midpoint of the greater curvature (arrows). Perigastric and mesenteric lymph nodes are enlarged (arrowheads). (C) Cytological findings (Intestines). Many macrophages contain bacterial organisms in their cytoplasm. The bacteria are unstained and rod or filamentous in appearance. Wright-Giemsa stain. (D) Intestines. There is severe multifocal to diffuse infiltration of inflammatory cells consisting of primarily macrophages and epithelioid cells with a small number of lymphocytes and neutrophils, throughout the lamina propria into the muscular layer. Wright-Giemsa. Bar=20 $\mu$ m (E) Intestines. Severe multifocal to diffuse infiltration of inflammatory cells comprised of predominantly macrophages and epithelioid cells with few lymphocytes and neutrophils was present in the lamina propria into the muscular layer. HE. Bar $=100 \mu \mathrm{m}$ (F) Ziehl-Neelsen stain. Macrophages and epithelioid cells contain intracytoplasmic filamentous pale to slightly basophilic bacteria, which are intensely positive for Ziehl-Neelsen stain. Bar=60 $\mu \mathrm{m}$ (G) Lymph node. Epithelioid macrophages containing acid fast bacterial organisms infiltrate diffusely in the sinus. HE. Bar=100 $\mu \mathrm{m}$ (H) Liver. Mild to moderate accumulation of macrophages, lymphocytes and a few neutrophils is noted around the portal area. The macrophages in the lesions also have acid-fast bacterial organisms in their cytoplasm. HE. Bar=100 $\mu \mathrm{m}$ 
generally effective for the treatment of most mycobacterium infections, whereas $M$. celatum is known to be resistant to rifampicin, and ciprofloxacin has been documented to be ineffective in the treatment of $M$. genavense. Additionally, the use of monotherapy with rifampicin generally results in the propensity for rapid development of resistance to this agent in vivo. In the present case, the patient's response to therapy remained unremarkable throughout treatment, so drug resistance may not have been a contributing cause for treatment failure, but drug choice or dosage was considered as possible factors for the poor prognosis. Therefore, it is proposed that identification of mycobacterial species is important, and characterization of data from additional cases is necessary.

With respect to side effects, the long-term administration of antimicrobial agents becomes problematic in regards to hepatotoxicity more so than bone marrow suppression. Similar to other reports, the mycobacterium infection in this case was disseminated within the stomach, abdominal lymph nodes and liver $[15,16,18]$. In this case, bone marrow aspiration was not performed, and it is unknown whether anemia and leukopenia were due to the disseminated mycobacterium infection or were side effects from long-term administration of antimicrobials. Similarly, the cause of icterus remains unknown, although mycobacterial organisms were identified in the liver.

Infections of the gastrointestinal tract are likely caused by oral ingestion of feed contaminated with mycobacterium $[2,16]$. Infection with $M$. abscessus might occur via a number of routes. M. abscessus is a ubiquitous saprophyte; thus, contamination of wounds by soil, inhalation of aerosolized particles of soil and ingestion of contaminated water are likely sources of infections [11]. Mycobacteria are more resistant to heat, $\mathrm{pH}$ changes and routine disinfectants than other pathogenic non-spore-forming bacteria [17]. In this case, the principal symptoms were related to only the gastrointestinal tract, and there were no obvious wounds or injuries on the body surface; therefore, the present case was presumed to be the result of infection by oral ingestion of the bacterium.

The relationship between mycobacteriosis and a weakened immune system is well-established in human medicine [12]. Immunosuppression has also been theorized to play a role in tuberculosis infections in the ferret. However, in previous cases $[13,18]$ and the present case, the affected ferrets did not appear to have underlying diseases or immunodeficiency disorders. Therefore, there is a risk for mycobacteriosis, even if patients are not immunocompromised. Moreover, the risk for $M$. celatum transmission from a ferret to a human was proposed in a German report [8]. The zoonotic risk of mycobacterial infections for immunocompromised as well as immunocompetent persons should be kept in mind, considering the possibility for reverse transmission and the close contact between animals and their owners, and it is necessary to pay sufficient attention to treating mycobacterial disease in animals in order to minimize this risk.

In conclusion, this paper describes the clinical course and outcome of a ferret infected with mycobacterium in Japan. The authors suggest that mycobacterium infection should be considered as a differential diagnosis for ferrets presenting with gastrointestinal symptoms.

\section{REFERENCES}

1. Bryant, J. L., Hanner, T. L., Fultz, D. G., Hurley, S. and BeschWilliford, C. 1988. A chronic granulomatous intestinal disease in ferrets caused by an acid-fast organism morphologically similar to Mycobacterium paratuberculosis. Lab. Anim. Sci. $\mathbf{3 8}$ : 498-499.

2. Cross, M. L., Labes, R. E. and Mackintosh, C. G. 2000. Oral infection of ferrets with virulent Mycobacterium bovis or Mycobacterium avium: susceptibility, pathogenesis and immune response. J. Comp. Pathol. 123: 15-21. [Medline] [CrossRef]

3. de Lisle, G. W., Crews, K., de Zwart, J., Jackson, R., Knowles, G. J., Paterson, K. D., MacKenzie, R. W., Waldrup, K. A. and Walker, R. 1993. Mycobacterium bovis infections in wild ferrets. N. Z. Vet. J. 41: 148-149. [Medline] [CrossRef]

4. de Lisle, G. W., Kawakami, R. P., Yates, G. F. and Collins, D. M. 2008. Isolation of Mycobacterium bovis and other mycobacterial species from ferrets and stoats. Vet. Microbiol. 132: 402-407. [Medline] [CrossRef]

5. Fox, J. G. 1998. Bacterial and Mycoplasmal diseases. pp. 343-347. In: Biology and Diseases of the Ferret, 2nd ed. (Fox, J.G. ed.), Williams and Wilkins, Baltimore.

6. Hoefer H. L., Fox, J. G. and Bell, J. A. 2012. Gastrointestinal diseases. pp. 27-45. In: Ferrets, Rabbits, and Rodents: Clinical Medicine and Surgery, 3rd ed. (Quesenberry, K.E. and Carpenter, J.W. eds.), W. B. Saunders, Philadelphia.

7. Lucas, J., Lucas, A., Furber, H., James, G., Hughes, M. S., Martin, P., Chen, S. C., Mitchell, D. H., Love, D. N. and Malik, R. 2000. Mycobacterium genavense infection in two aged ferrets with conjunctival lesions. Aust. Vet. J. 78: 685-689. [Medline] [CrossRef]

8. Ludwig, E., Reischl, U., Holzmann, T., Melzl, H., Janik, D., Gilch, C. and Hermanns, W. 2011. Risk for Mycobacterium celatum infection from ferret. Emerg. Infect. Dis. 17: 553-555. [Medline] [CrossRef]

9. Lugton, I. W., Wobeser, G., Morris, R. S. and Caley, P. 1997. Epidemiology of Mycobacterium bovis infection in feral ferrets (Mustela furo) in New Zealand: I. Pathology and diagnosis. N. Z. Vet. J. 45: 140-150. [Medline] [CrossRef]

10. Lugton, I. W., Wobeser, G., Morris, R. S. and Caley, P. 1997. Epidemiology of Mycobacterium bovis infection in feral ferrets (Mustela furo) in New Zealand: II. Routes of infection and excretion. N. Z. Vet. J. 45: 151-157. [Medline] [CrossRef]

11. Lunn, J. A., Martin, P., Zaki, S. and Malik, R. 2005. Pneumonia due to Mycobacterium abscessus in two domestic ferrets (Mustelo putorius furo). Aust. Vet. J. 83: 542-546. [Medline] [CrossRef]

12. Orcau, À., Caylà, J. A. and Martínez, J. A. 2011. Present epidemiology of tuberculosis. Prevention and control programs. Enferm. Infecc. Microbiol. Clin. 29: 2-7. [Medline] [CrossRef]

13. Piseddu, E., Trotta, M., Tortoli, E., Avanzi, M., Tasca, S. and Solano-Gallego, L. 2011. Detection and molecular characterization of Mycobacterium celatum as a cause of splenitis in a domestic ferret (Mustela putorius furo). J. Comp. Pathol. 144: 214-218. [Medline] [CrossRef]

14. Pollock, C. 2012. Mycobacterial infection in the ferret. Vet. Clin. Exot. Anim. 15: 121-129. [Medline] [CrossRef]

15. Saunders, G. K. and Thomsen, B. V. 2006. Lymphoma and Mycobacterium avium infection in a ferret (Mustela putorius furo). Vet. Clin. North Am. Exot. Anim. Pract. 18: 513-515. [Medline] 
16. Schultheiss, P. C. and Dolginow, S. Z. 1994. Granulomatous enteritis caused by Mycobacterium avium in a ferret. J. Am. Vet. Med. Assoc. 204: 1217-1218. [Medline]

17. Tiwari, T. S., Ray, B., Jost, K. C. Jr., Rathod, M. K., Zhang, Y., Brown-Elliott, B. A., Hendricks, K. and Wallace, R. J. Jr. 2003. Forty years of disinfectant failure: outbreak of postinjection Mycobacterium abscessus infection caused by contamination of benzalkonium chloride. Clin. Infect. Dis. 36: 954-962. [Medline] [CrossRef]
18. Valheim, M., Djønne, B., Heiene, R. and Caugant, D. A. 2001. Disseminated Mycobacterium celatum (type 3) infection in a domestic ferret (Mustela putorius furo). Vet. Pathol. 38: 460-463. [Medline] [CrossRef]

19. Vrioni, G., Nauciel, C., Kerharo, G. and Matsiota-Bernard, P. 1998. Treatment of disseminated Mycobacterium genavense infection in a murine model with ciprofloxacin, amikacin, ethambutol, clarithromycin and rifabutin. J. Antimicrob. Chemother. 42: 483-487. [Medline] [CrossRef] 